\title{
Faits marquants en spintronique depuis la découverte de la magnétorésistance géante
}

Bernard Dieny (bernard.dieny@cea.fr)

SPINTEC, UMR 8191 CEA/CNRS/UJF, Institut Nanosciences et Cryogénie, CEA/Grenoble, 38054 Grenoble Cedex 9

La découverte de la magnéto-

résistance géante (GMR) par

Albert Fert et Peter Grünberg

est considérée comme fondatrice

de l'électronique de spin.

Celle-ci réalise un mariage

entre l'électronique et

le magnétisme. Cette discipline

a maintenant une vingtaine

d'années. Depuis 1988,

son essor a été stimulé

par une série de découvertes

marquantes relatées dans

cet article : magnétorésistance

tunnel (TMR), transfert de spin,

dispositifs hybrides magnétiques/

semi-conducteurs.

Ces découvertes ont permis

divers développements

applicatifs dans les domaines

des mémoires non volatiles

(MRAM), des composants

pour la logique, des composants

radiofréquences pour

les télécommunications,

et des capteurs de champs

magnétiques hypersensibles.
L'article d'Albert Fert (Reflets de la physique $\mathrm{n}^{\circ} 15$, pp. 5-10) a parfaitement introduit la thématique de l'électronique de spin et le mécanisme de la magnétorésistance géante. De manière simple, on peut dire que les matériaux magnétiques peuvent servir de polariseurs ou d'analyseurs d'électrons polarisés en spin. La spintronique associe ainsi des matériaux magnétiques et non magnétiques, ces matériaux pouvant être métalliques, isolants ou semi-conducteurs. Les composants spintroniques ont souvent une structure de la forme couche ferromagnétique (F1)/couche non magnétique (NM)/couche ferromagnétique (F2), dans laquelle on peut changer l'orientation relative des aimantations des couches F1 et F2. Cette structure s'apparente à un couple polariseur/ analyseur en optique. En optique, il est connu qu'en jouant sur l'angle entre les directions de polarisation du polariseur et de l'analyseur, on peut changer la transmission de la lumière à travers le couple polariseur/analyseur. En électronique de spin, lorsqu'un courant électrique traverse la structure F1/NM/F2 perpendiculairement aux interfaces (GMR en géométrie CPP, voir l'article d'A. Fert

\section{Les vannes de spin}

Très rapidement après la découverte de la magnétorésistance géante, les industriels de l'enregistrement magnétique ont perçu que ce nouveau phénomène pouvait être très utile pour la réalisation de capteurs de champ magnétique ultrasensibles, en particulier pour les têtes de lecture de disques durs d'ordinateurs.

Toutefois, dans les multicouches $(\mathrm{Fe} / \mathrm{Cr}$ ) étudiées par les équipes d'A. Fert et P. Grünberg, les champs nécessaires pour observer la magnétorésistance géante étaient très élevés, typiquement 2 teslas, du fait de l'existence d'un fort couplage antiferromagnétique entre les aimantations des couches de fer à travers les couches de chrome. Or, le champ magnétique que l'on cherche à relire à la surface des disques durs pour connaitre l'information écrite dans dans Reflets $n^{\circ} 15$, p. 9), la première couche ferromagnétique (F1) polarise en spin les électrons parallèlement à l'aimantation de cette couche. Cette polarisation est transmise à travers la couche non magnétique supposée suffisamment fine (typiquement quelques nanomètres). La capacité des électrons à pénétrer dans la couche F2 dépend alors de l'angle entre les aimantations de F1 et F2. Ceci se manifeste par un changement de la résistance électrique de la structure en fonction de cet angle.

Ce phénomène a été mis en évidence pour la première fois à Orsay en 1988 dans des multicouches constituées d'alternance de couches de fer de $2 \mathrm{~nm}$ et de chrome de $1 \mathrm{~nm}$ [1]. Il lui a été donné le nom de magnétorésistance géante, car le changement relatif de résistance observé sous champ magnétique, de l'ordre de 50\% à température ambiante, était beaucoup plus grand que tous les phénomènes de magnétorésistance connus jusque-là dans les métaux. Cette découverte est considérée comme fondatrice de l'électronique de spin. Elle a stimulé une foison d'études sur ces phénomènes d'interaction entre magnétisme et transport

le disque est de l'ordre de 2 à 4 millitesla, soit 3 ordres de grandeur plus faible. Il fallait donc mettre au point des matériaux présentant les effets de magnétorésistance géante à champs beaucoup plus faibles. Ceci a été réalisé à IBM Almaden par l'équipe de V. Speriosu [2], par le développement des "vannes de spin ».

Les vannes de spin sont essentiellement constituées de deux couches magnétiques quasiment totalement découplées, séparées par une couche non magnétique, par exemple en cuivre, d'épaisseur supérieure à $2 \mathrm{~nm}$ (fig. A) . Afin de pouvoir varier l'orientation relative des aimantations des deux couches ferromagnétiques, I'aimantation d'une de ces couches (dite piégée) est couplée par interaction d'échange avec une couche antiferromagnétique (dite de piégeage). 
de charge. En 1990, des matériaux présentant une magnétorésistance géante à champ faible, les "vannes de spin" (voir encadré, ci dessous), ont été mis au point pour réaliser des capteurs ultrasensibles de champs magnétiques [2]. Ces capteurs ont été introduits dans les têtes de lecture des disques durs d'ordinateurs en 1998. Ce fut la première application industrielle de composants spintroniques.

Depuis, l'électronique de spin a connu plusieurs découvertes marquantes, qui vont être décrites dans cet article :

- observation en 1995 de magnétorésistance tunnel dans des jonctions tunnel magnétiques à température ambiante, d'abord sur des jonctions amorphes (donnant des amplitudes de magnétorésistance de l'ordre de $50 \%$ ), puis sur des jonctions cristallisées (barrière tunnel de $\mathrm{MgO}$ donnant des amplitudes de magnétorésistance jusqu'à $600 \%)$;

- prédiction (1996) puis observation expérimentale (1998, 2000) d'effets de transfert de spin, permettant de manipuler l'aimantation de nanostructures magnétiques par des courants polarisés en spin;

》〉

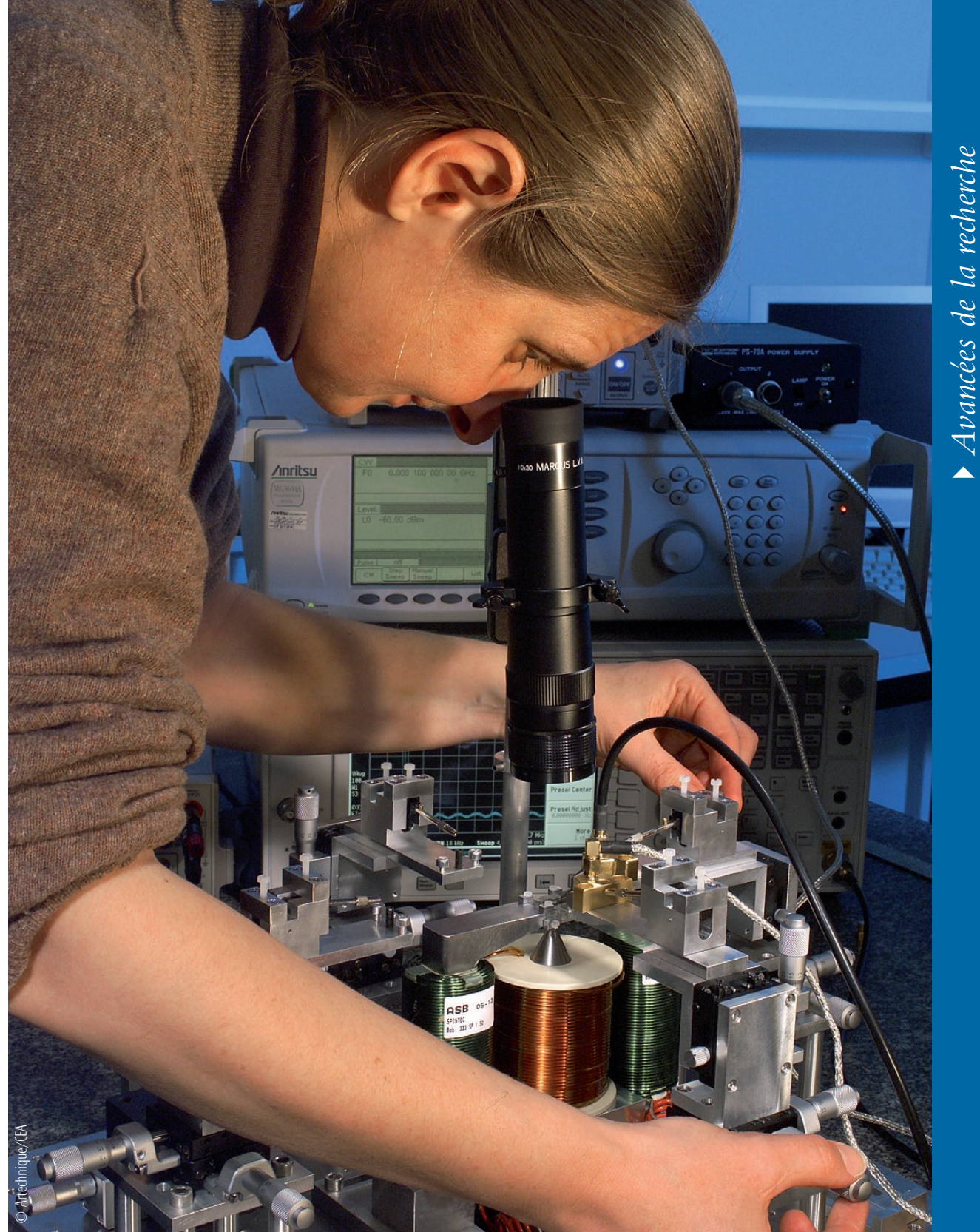

Banc de mesures électriques spectrales large bande $(0-26 \mathrm{GHz})$ dédié à l'étude de nanostructures magnétiques à courant polarisé en spin au CEA/Grenoble.
L'interaction à travers l'interface entre ces deux couches a pour effet de bloquer l'aimantation de la couche piégée dans une direction fixe sur une très large gamme de champ appliqué.
Par contre, l'autre couche magnétique, dite " libre », est constituée d'un matériau magnétiquement doux, c'est-à-dire dont l'aimantation s'oriente très facilement dans le champ appliqué,

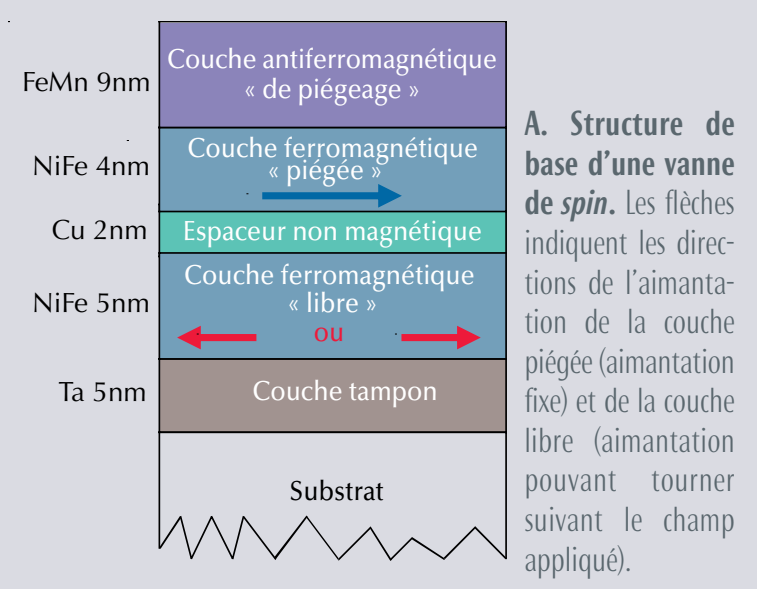

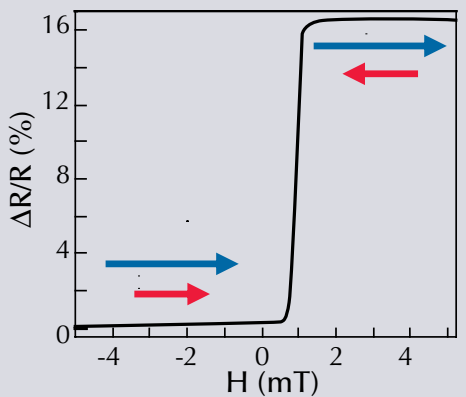

B. Réponse magnétorésistive d'une vanne de spin. Les flèches indiquent les directions d'aimantation des couches libre (flèches rouges) et piégée (flèches bleues). comme une aiguille de boussole. Un faible champ magnétique suffit alors à provoquer une variation d'orientation relative d'aimantation et donc l'effet de GMR (fig. B).

L'optimisation de ces vannes de spin entre 1990 et 1998, jusqu'à leur introduction dans les disques durs, a conduit à l'essor de toute une ingénierie de multicouches magnétiques pour la spintronique. Les structures optimisées comprennent aujourd'hui une vingtaine de couches, dont les épaisseurs sont contrôlées à I'angström près. La réponse, très linéaire et réversible, observée sur la figure $B$, montre que ces matériaux sont tout à fait adaptés pour la réalisation de capteurs de champs magnétiques très sensibles. 


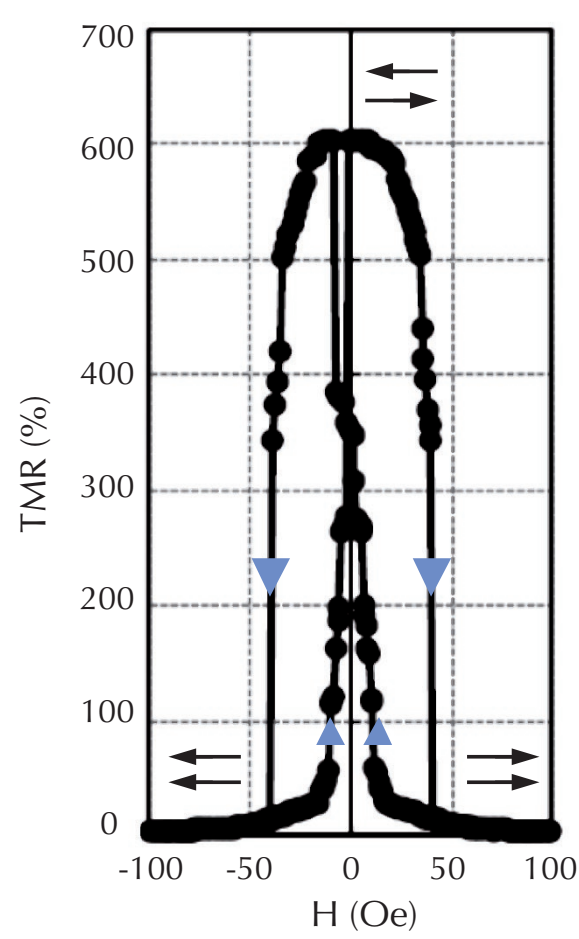

1. Magnétorésistance à température ambiante de jonctions tunnel à base de $\mathrm{Mg} 0$, d'après Yuasa et al., Appl. Phys. Lett. 89 (2006) 042505. La résistance de l'échantillon varie de $4 \mathrm{k} \Omega$ à $17 \mathrm{k} \Omega$ lorsque les aimantations des deux électrodes magnétiques passent d'un alignement parallèle à un alignement antiparallèle. Les flèches bleues indiquent le sens de parcours du cycle de magnétorésistance. L'amplitude de TMR est définie comme la variation relative de la résistance de la jonction tunnel entre les configurations magnétiques parallèle $\left(R_{p}\right)$ et antiparallèle $\left(R_{A P}\right): T M R=\left(R_{A P}-R_{P}\right) / R_{p}$. L'hystérésis observée sur la magnétorésistance résulte de l'hystérésis dans le retournement de l'aimantation des deux électrodes magnétiques de la jonction.
- possibilité de modifier les propriétés magnétiques par tensions électriques (2000). Dans ce domaine, les semi-conducteurs magnétiques jouent un rôle très important. Cette partie sera traitée plus en détail dans l'article d'Agnès Barthélémy et al. (à paraître dans Reflets $\mathrm{n}^{\circ} 17$ ).

\section{Magnétorésistance tunnel}

Les jonctions tunnel magnétiques se présentent aussi sous la forme de sandwiches F1/NM/F2 mais, à la différence des vannes de spin dans lesquelles la couche séparatrice NM est métallique (typiquement en cuivre), la couche séparatrice est ici une fine couche de matériau isolant (par exemple en alumine d'épaisseur 1,5 nm). Cette couche constitue une barrière d'énergie pour les électrons de conduction. Ceux-ci peuvent néanmoins traverser la barrière par effet tunnel. Lorsque les électrodes de part et d'autre de la barrière tunnel sont magnétiques, la probabilité de passage des électrons à travers cette barrière dépend de l'angle entre le spin des électrons et l'aimantation de l'électrode qui reçoit l'électron après passage de la barrière. De ce fait, lorsqu'une tension électrique est appliquée entre les deux électrodes de la jonction, le courant d'électrons à travers celle-ci dépend de l'angle entre les aimantations des deux électrodes. Cet effet avait été observé pour la première fois en 1975 par un Français, Michel Jullière, à basse température dans des jonctions magnétiques semi-conductrices à base de germanium [3]. Mais l'intérêt pour les jonctions tunnel n'a vraiment explosé qu'après l'observation en 1995 de magnétorésistance tunnel à température ambiante dans des jonctions amorphes à base d'alumine [4]. Ces jonctions présentaient des amplitudes de magnétorésistance de 30 à 70\%. Depuis, d'énormes progrès ont été réalisés dans la nature et la qualité des matériaux constituant les barrières tunnel. En particulier, des jonctions magnétiques cristallisées ont été mises au point, à base d'oxyde de magnésium ( $\mathrm{MgO})$, présentant des amplitudes de magnétorésistance supérieures à $600 \%$ à température ambiante (fig. 1), donc très supérieures à celles observées avec la magnétorésistance géante classique.

Cette augmentation considérable de l'amplitude de la magnétorésistance des jonctions à base de $\mathrm{MgO}$ par rapport aux jonctions amorphes à base d'alumine provient de la nature cristallisée des barrières de $\mathrm{MgO}$. Dans les jonctions à barrières amorphes, la magnétorésistance provient uniquement de la différence de population en spin $\uparrow$ et $\downarrow$ dans l'électrode magnétique au voisinage immédiat de la barrière tunnel. Dans les jonctions tunnel cristallisées, un phénomène supplémentaire se produit : il s'agit d'un filtrage des électrons qui passent à travers la barrière tunnel, suivant la symétrie de leur fonction d'onde électronique. Seuls les électrons dont la fonction d'onde a une symétrie compatible avec celle du réseau cristallographique de $\mathrm{MgO}$, arrivent à passer à travers la barrière tunnel. Or, si les électrodes sont en alliages riches en cobalt de structures cubiques comme le $\mathrm{MgO}$, seuls les électrons de spin $\uparrow$ ont la symétrie adéquate pour passer à travers la barrière tunnel. Il en résulte un filtrage quasi-parfait des électrons $\uparrow$, donc une polarisation des électrons tunnel proche de $100 \%$, d'où de très fortes amplitudes de magnétorésistance.

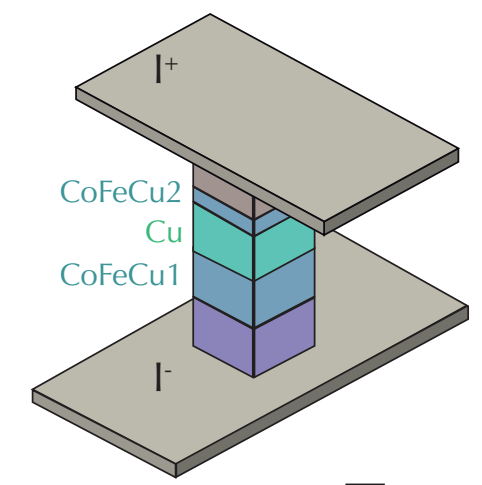

$\mathrm{a}$
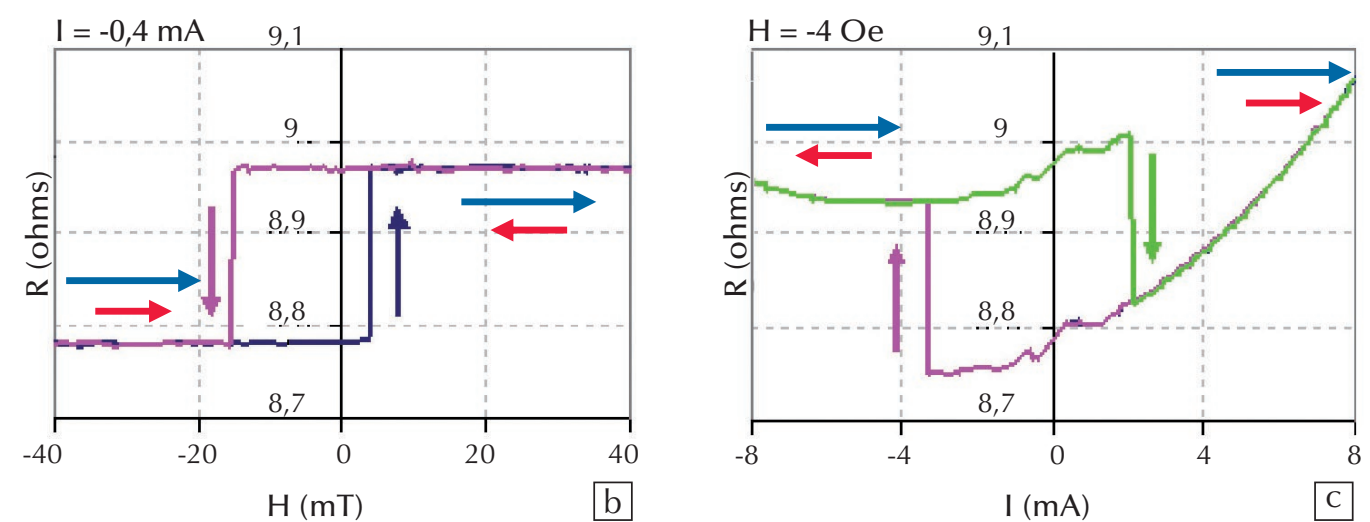

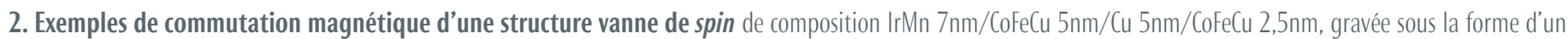
pilier de section $130 \mathrm{~nm}$ x $130 \mathrm{~nm}$ et traversée par un courant perpendiculairement au plan des couches (a). Dans la figure du centre (b), la commutation est provoquée par l'application d'un champ magnétique parallèle au plan des couches, qui fait commuter l'aimantation de la couche libre (CoFeCu 2,5nm) de la vanne de spin de façon hystérétique. Les flèches mauve et indigo indiquent le sens de parcours des cycles d'hystérésis. Le changement de résistance observé est dû à la magnétorésistance géante de la structure. Dans la figure de droite (c), la commutation est provoquée par le courant polarisé en spin, circulant à travers la structure (d’après [8]). La courbure asymétrique observée sur les courbes $R(I)$ est liée à une combinaison d'effets Joule et thermoélectrique se produisant dans la nanostructure. 
Outre l'intérêt de ces phénomènes du point de vue fondamental, les jonctions tunnel suscitent un intérêt considérable pour différents types d'applications mémoires, composants logiques ou capteurs, qui seront décrits dans les articles ultérieurs de ce dossier.

\section{Transfert de spin}

Les phénomènes d'électronique de spin dont nous avons parlé jusqu'ici sont des phénomènes de magnétorésistance (géante GMR ou tunnel TMR), c'est-à-dire des variations de résistance électrique sous champ magnétique. Ces effets permettent d'agir sur un courant électrique polarisé en spin à partir de l'aimantation d'une nanostructure magnétique. Mais l'effet réciproque existe aussi. Il a été prédit en 1996 par deux théoriciens : J. Slonczewski et L. Berger [5], et a été observé expérimentalement peu de temps après [6,7]. Cet effet, appelé "transfert de spin ", est une action qu'exerce un courant polarisé en spin sur l'aimantation d'une nanostructure. L'idée est la suivante : lorsque des électrons polarisés en spin sont injectés dans une nanostructure magnétique dont l'aimantation fait un angle avec la direction de polarisation en spin des électrons, cette dernière se réoriente très rapidement parallèlement à l'aimantation locale dans le premier nanomètre suivant la pénétration des électrons dans la nanostructure. En réaction, cette réorientation se traduit par un couple magnétique agissant sur l'aimantation de la nanostructure. Ce couple peut produire différents effets.

a) Il peut faire commuter l'aimantation de la nanostructure dans une direction ou la direction opposée, suivant le sens du courant. Ceci est illustré à titre d'exemple sur la figure 2. Les expériences ont d'abord été réalisées sur des structures de type vannes de spin métalliques, comme celles utilisées dans la figure 2. Les densités de courant critique nécessaires pour provoquer la commutation d'aimantation dans ces structures métalliques sont de quelques $10^{7} \mathrm{~A} / \mathrm{cm}^{2}$.

Ces fortes densités de courant interdisaient au départ d'observer ce type d'effet dans les jonctions tunnel magnétiques, car celles-ci sont sujettes au claquage électrique lorsque la tension qui leur est appliquée est trop importante (typiquement supérieure à $\sim 1 \mathrm{~V})$. Toutefois, grâce aux progrès réalisés dans la croissance des jonctions tunnel, il est devenu possible d'abaisser suffisamment l'épaisseur des barrières tunnel et donc leur transparence (mesurée par leur produit $\mathrm{RA}=$ résistance $\times$ surface, la surface étant l'aire de la jonction), pour observer ces effets de commutation dans les jonctions tunnel [9].

b) Dans certaines géométries, le champ appliqué et le transfert de spin peuvent exercer des influences antagonistes sur l'aimantation d'une nanostructure. Dans ce cas, le transfert de spin peut générer des excitations entretenues de l'aimantation. Le système pompe constamment de l'énergie au courant polarisé en spin pour compenser les mécanismes de dissipation spin-réseau. Par exemple, il a été montré que l'injection d'électrons polarisés en spin hors du plan dans une couche magnétique à aimantation planaire permet de générer un mouvement de précession entretenue de l'aimantation de cette couche sur un cône d'axe perpendiculaire au plan (fig. 3a). La fréquence de précession dépend de la densité du courant continu traversant la structure. $\mathrm{Si}$ on complète la structure par une couche de référence d'aimantation fixe, la variation oscillante d'angle entre l'aimantation excitée et l'aimantation fixe provoque une variation sinusoïdale de résistance, se traduisant elle-même par une oscillation de tension aux bornes du dispositif. La fréquence de ces oscillations se situe dans la gamme 2-30 GHz et peut être ajustée par le courant traversant la structure (fig. 3b).

c) Le transfert de spin peut également se manifester comme une action, provoquée par la circulation d'un courant électrique, sur les parois magnétiques existant dans un matériau magnétique homogène. $\mathrm{Ce}$ phénomène a été prédit initialement par Berger en 1984 [11] et observé une vingtaine d'années plus tard dans des nanofils magnétiques de différents types de matériaux semi-conducteurs, métalliques ou oxydes. L'idée générale est la suivante. Si on considère par exemple une nanobande de $\mathrm{NiFe}$ dont l'aimantation est planaire, cette aimantation a tendance à s'aligner avec la longueur de la nanobande (fig. 4), pour des raisons d'énergie magnétostatique. Il se forme alors, le long de la bande, des domaines magnétiques, c'est-à-dire des régions d'aimantation quasi-uniforme, séparées les unes des autres par des parois. Les parois sont des zones de rotation d'aimantation, dont la forme résulte d'un compromis entre énergies d'échange, d'anisotropie et magnétostatique. Les parois peuvent avoir différentes configurations. Si la nanobande est étroite, la paroi sera plutôt de type transverse, comme représenté sur la figure 4.

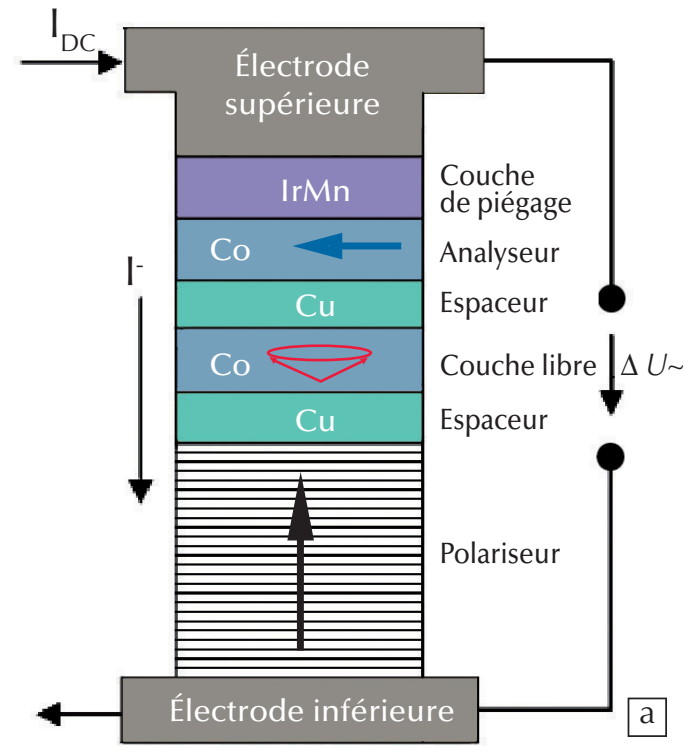

Électrode inférieure
I $>0$

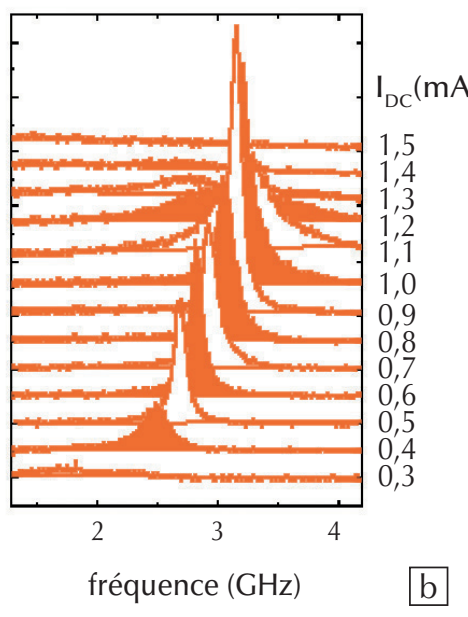

3. (a) Schéma d'un oscillateur spintronique à polariseur perpendiculaire au plan des couches. L'oscillateur est un pilier légèrement elliptique de dimension $60 \mathrm{~nm}$ x $70 \mathrm{~nm}$ et de composition Polariseur $/ \mathrm{Cu} / \mathrm{C}_{0} / \mathrm{Cu} / \mathrm{C}_{0} / \mathrm{lrMn}$, où le polariseur perpendiculaire est lui-même une multicouche à anisotropie hors du plan. Le courant continu traversant la structure entretient un mouvement de précession de l'aimantation de la couche dite « libre " (Co) sur un cône d'axe perpendiculaire au plan de la couche et parallèle à la direction de polarisation des spins du courant. L'analyseur est une couche de Co dont l'aimantation est piégée par interaction d'échange avec la couche antiferromagnétique IrMn (voir l'encadré sur les vannes de spin, pp. 6-7). Via l'effet de magnétorésistance lié à la variation d'angle entre les aimantations des couches libre et piégée (analyseur), une tension alternative $\Delta U \sim$ apparait entre les deux électrodes du dispositif.

(b) Spectres expérimentaux de la densité spectrale de la tension de sortie $\Delta U \sim$ pour différents courants variant entre 0,3 et 1,5 mA. La fréquence des oscillations augmente avec le courant. (D'après [10]). 
Lorsqu'un courant circule le long de la nanobande, les électrons acquièrent une polarisation en spin en se propageant à l'intérieur d'un domaine. Lorsqu'un électron traverse la paroi, son spin peut se réorienter continûment au sein de la paroi et accompagner la rotation du champ dans la paroi (on parle de transmission adiabatique du spin) ou, à l'autre extrême, peut être transmis d'un domaine à l'autre à travers la paroi sans subir de réorientation (transmission non adiabatique). Il a été montré théoriquement et expérimentalement qu'une transmission purement adiabatique du spin conduit essentiellement à une déformation de la paroi, alors qu'une transmission non adiabatique exerce une pression sur la paroi qui peut la dépiéger d'un défaut et la mettre en mouvement. Les densités de courant auxquelles ces effets sont observés sont de l'ordre de $10^{8} \mathrm{~A} / \mathrm{cm}^{2}$ dans les systèmes magnétiques métalliques, mais seulement de l'ordre de $10^{5}$ à $10^{6} \mathrm{~A} / \mathrm{cm}^{2}$ dans les semi-conducteurs ou oxydes magnétiques.

Ces différents phénomènes de transfert de spin suscitent un intérêt considérable, car ils ouvrent tout un nouveau champ d'étude de la dynamique d'aimantation induite par un courant polarisé en spin. Ils ouvrent par ailleurs de nouvelles voies de manipulation de l'aimantation de nanostructures magnétiques, et en particulier d'écriture d'information dans des cellules

\section{Biblioaraphie}

Pour en savoir plus sur la spintronique, on consultera les 9 articles réunis par A. Fert et J.P. Nozières dans les Comptes Rendus Physique, Tome 6, fascicule 9 (novembre 2005).

1 M. Baibich et al., Phys. Rev. Lett. 61 (1988) 2472.

2 B. Dieny, V.S. Speriosu et al., Phys. Rev. B 43 (1991) 1297-1300.

3 • M. Jullière, Physics Letters A 54 (1975) 225.

4 • J.S. Moodera et al., Phys. Rev. Lett. 74 (1995) 3273-3276 ; T. Miyazaki et N. Tezuka, J. Magn. Magn. Mater. 139 (1995) L231.

5 • J. Slonczewski, J. Magn. Magn. Mater. 159 (1996) L1 L. Berger, Phys. Rev. B 54 (1996) 9353.

6 - M. Tsoi et al., Phys. Rev. Lett. 80 (1998) 4281.

7 • J.A. Katine et al., Phys. Rev. Lett. 84 (2000) 3149.

8 • A. Deac et al., Phys. Rev. B 73 (2006) 064414.

9 •. Huai et al., Applied Physics Letters 84 (2004) 3118.

10 - D. Houssameddine et al., Nature Materials 6 (2007) 447.

11 - L. Berger, Journ. Appl. Phys. 55 (1984) 1954.

12 • S. Datta et B. Das, Applied Phys. Lett. 56 (1990) 665.

13 - X. Jiang et al., Phys. Rev. Lett. 94 (2005) 056601.

14 - D. Chiba et al., Science 301 (2003) 943.

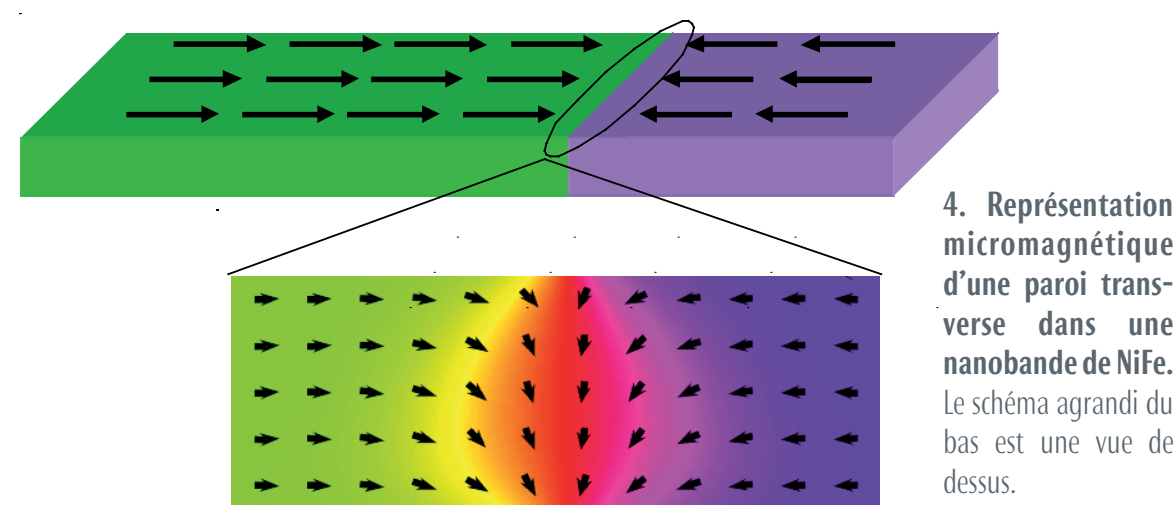

mémoires ou de génération d'excitations entretenues d'aimantation pour des oscillateurs radiofréquence, ou encore de mouvements contrôlés de parois magnétiques pour la réalisation de mémoires de masse.

\section{Dispositifs hybrides magnétiques/semi-conducteurs}

L'utilisation de semi-conducteurs en électronique de spin offre une très grande richesse de phénomènes, chacun faisant l'objet de nombreuses études. Les semi-conducteurs sont des matériaux de choix pour l'électronique de spin pour plusieurs raisons.

- Dans les semi-conducteurs de type IV (silicium, germanium), le couplage spinorbite est faible (particulièrement pour le silicium), ce qui permet, si on polarise en spin les porteurs de charge à un certain endroit du semi-conducteur, de conserver cette polarisation en spin sur une longue distance (pouvant atteindre plusieurs microns dans le silicium). Ceci permet de concevoir des dispositifs dans lesquels on injecte dans le semi-conducteur des électrons polarisés en spin au niveau d'une première électrode, on les manipule lors de leur propagation dans le semiconducteur (par des champs magnétiques ou des champs électriques), et on les collecte de façon sélective en spin au niveau d'une deuxième électrode. Un transistor de spin fonctionnant sur ce principe a été proposé de façon conceptuelle par Datta et Das en 1990 [12].

- Par ailleurs, les semi-conducteurs (particulièrement les III-V et les II-VI) permettent de convertir des effets optiques en effets électroniques ou réciproquement. En irradiant un semi-conducteur par une lumière polarisée en spin circulairement, on peut générer (par le jeu des règles de sélection) des paires électrons-trous dans lesquelles les électrons sont polarisés en spin. Inversement, lors de la recombinaison radiative d'un électron polarisé en spin et d'un trou, on peut émettre un photon de polarisation circulaire. C'est d'ailleurs ainsi que l'efficacité de l'injection d'électrons polarisés en spin dans un semi-conducteur est souvent mesurée. Par exemple dans la référence [13], l'injecteur $\mathrm{CoFe} / \mathrm{MgO}$ était déposé sur une diode photoluminescente (LED) à base de puit quantique GaAs/AlGaAs/GaAs. La mesure de la polarisation circulaire de la lumière émise par la spin-LED renseigne directement sur la polarisation en spin des électrons injectés dans le semi-conducteur.

- Enfin, une des grandes caractéristiques des semi-conducteurs est de permettre de moduler la densité de porteurs de charge par l'application de tension électrique. Ceci offre des possibilités inédites de contrôle des propriétés magnétiques de composants spintroniques par des tensions électriques (et non par le courant), ce qui a été montré par exemple dans $\operatorname{In}_{1-\mathrm{x}} \mathrm{Mn}_{\mathrm{x}} \mathrm{As}$ par le groupe de $\mathrm{H}$. Ohno à l'université de Tohoku (Japon) [14] (fig. 5). Dans ce type de semi-conducteur dopé avec des ions magnétiques de manganèse, le caractère ferromagnétique provient des interactions d'échange entre ions $\mathrm{Mn}$. Ces interactions sont en fait transmises par les porteurs de charge qui se polarisent au voisinage d'un ion $\mathrm{Mn}$, puis transportent cette polarisation aux ions $\mathrm{Mn}$ voisins. Mais on comprend aisément que dans ce mécanisme d'échange, l'intensité de l'interaction dépend directement de la densité de porteurs de charge. Ainsi, en contrôlant cette densité par une tension électrique, on modifie l'intensité des interactions d'échange et donc la température d'ordre ferromagnétique (la température de Curie Tc) du matériau. Dans les matériaux magnétiques, le rapport $\mathrm{T} / \mathrm{Tc}$ joue un rôle très important, car il caractérise l'activation thermique de l'aimantation. 
En modifiant la tension appliquée, on change cette activation thermique comme si on variait la température du système. Ceci peut conduire à des transitions ferromagnétique/paramagnétique ou à des changements de coercitivité induits par tension (voir l'article d'Agnès Barthélémy et al., dans Reflets ${ }^{\circ} 17$ ).

\section{Conclusion}

L'électronique de spin est une discipline en pleine croissance. Elle a été stimulée initialement par les développements dans le domaine de l'enregistrement magnétique à très haute densité (disques durs). Mais dans son essor, plusieurs découvertes marquantes ont ouvert de nouveaux champs d'investigation et d'applications. Parmi celles-ci, les effets de magnétorésistance tunnel à température ambiante et de transfert de spin ont été des étapes particulièrement

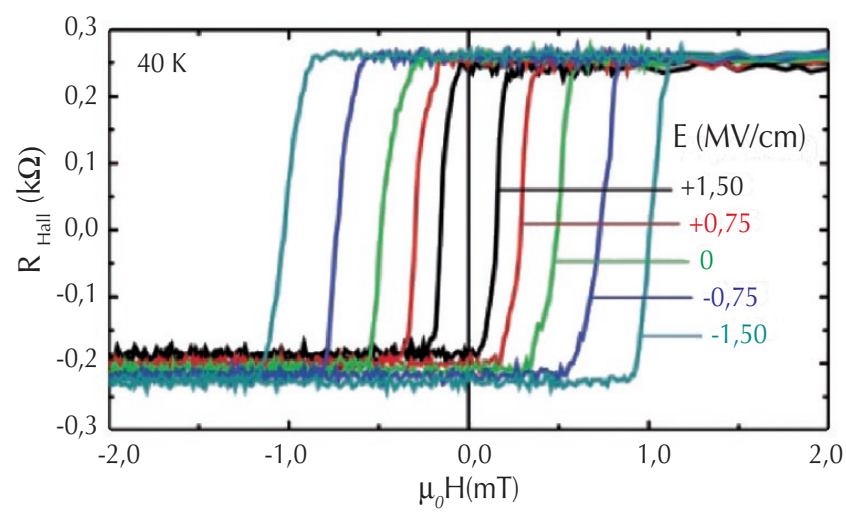

5. Modification de la coercitivité du semi-conducteur magnétique $\quad I n_{1-x} M n_{x} A s$ par l'application de tension électrique à l'aide d'une électrode de grille placée au-dessus d'une croix de Hall (d'après [14]).

significatives. Les perspectives d'applications se sont diversifiées : mémoires non volatiles (MRAM) (voir l'article de Jean-Pierre Nozières, à paraître dans Reflets $\left.\mathrm{n}^{\circ} 18\right)$, logique non volatile, logique reprogrammable, composants radiofréquences... La France est bien placée du point de vue recherche amont dans le domaine (Prix
Nobel d'Albert Fert en 2007 !), des start-up françaises apparaissent sur ces thématiques. Il est fort possible qu'après avoir disparu du domaine de l'enregistrement magnétique, une industrie du magnétisme arrive à se reconstruire en France et en Europe autour de l'électronique de spin. 